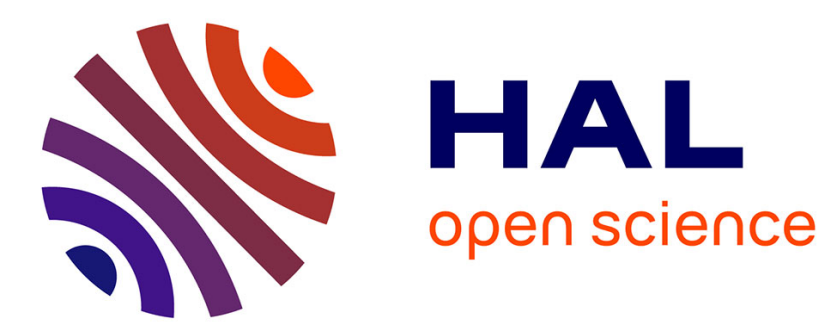

\title{
Manifolds in random media: two extreme cases
}

M. Mézard, G. Parisi

\section{To cite this version:}

M. Mézard, G. Parisi. Manifolds in random media: two extreme cases. Journal de Physique I, 1992, 2 (12), pp.2231-2242. 10.1051/jp1:1992278 . jpa-00246698

\section{HAL Id: jpa-00246698 https://hal.science/jpa-00246698}

Submitted on 1 Jan 1992

HAL is a multi-disciplinary open access archive for the deposit and dissemination of scientific research documents, whether they are published or not. The documents may come from teaching and research institutions in France or abroad, or from public or private research centers.
L'archive ouverte pluridisciplinaire HAL, est destinée au dépôt et à la diffusion de documents scientifiques de niveau recherche, publiés ou non, émanant des établissements d'enseignement et de recherche français ou étrangers, des laboratoires publics ou privés. 
Classification

Physics Abstracts

$05.40-75.10 \mathrm{~N}$

\title{
Manifolds in random media: two extreme cases
}

\author{
M. Mézard $\left({ }^{1}\right)$ and G.Parisi $\left({ }^{2}\right)$
}

( $\left.{ }^{1}\right)$ Laboratoire de Physique Théorique, Ecole Normale Supérieure( $\left.{ }^{*}\right) 24$ Rue Lhomond, 75231 Paris Cedex 05, France

( ${ }^{2}$ ) Dipartimento di fisica, Università di Roma II, via E. Carnevale, Roma 00173, Italy

(Received 27 May 1992, accepted 7 September 1992)

Résumé. - Nous étudions deux cas particuliers de variétés dirigées en milieu aléatoire. L'un est le modèle à zéro dimension d'une particule dans un potentiel aléatoire, l'autre le cas limite où la variété est plongée dans un espace de dimension élevée. Nous utilisons la méthode introduite récemment des variations gaussiennes dans l'espace à des répliques, avec brisure de la symétrie des répliques, et nous la comparons à des résultats connus ainsi qu'à des simulations numériques de ce modèle. En grande dimension $(N \rightarrow \infty)$ la méthode variationnelle est exacte. Nous calculons un des diagrammes qui contribuent aux corrections en $1 / N$.

\begin{abstract}
We discuss two special cases of directed manifolds in random media. One is the zero dimensional "toy" model of one particle in a random potential, the other is the limit where the manifold is embedded in a space of large dimensionality. We use the recently introduced Gaussian variational approach in replica space, with replica symmetry breaking, and compare it to known results and simulations of the toy model. In large dimensions $(N \rightarrow \infty)$ the variational approach is exact. We compute one of the diagrams contributing to the $O(1 / N)$ correction.
\end{abstract}

\section{Introduction.}

In the presence of quenched disorder, equilibrium statistical mechanics - or field theories present some interesting new phenomena which are not easy to study analytically. Systems like polymers or manifolds in random media, the random field Ising model etc.., present many metastable states which cannot be handled by standard perturbation theory. Last year there had been proposed a combination of the replica approach with a variational method of the Hartree Fock type, in order to study such systems [1]. The existence of the many metastable states is then taken care of by a spontaneous breakdown of replica symmetry (r.s.b.).

Recently this method has been extended to the study of the flux line lattice in high $T_{c}$ superconductors [2,3], and to the random field Ising model in the bulk [4]. In this paper we

(*) Unité propre du CNRS, associée à l'Ecole Normale Supérieure et à l'Université de Paris Sud. 
apply it to two extreme cases of the random manifold problem: the zero dimensional case of one particle in a random potential, which is a toy model of disordered systems, on which the general approach is tested and compared to standard approaches. The other case is that of a manifold in a space of infinite dimension $(N \rightarrow \infty)$. In this limit the approach of [1] is exact, and gives back the Flory like exponent for the wandering of the manifold. We carry here the first step of an expansion to order $1 / N$, by an explicit computation of the simplest $O(1 / N)$ diagram of the self energy. Although this correction looks divergent at first sight, we will show how a special cancellation due to some "replica identities" make this correction finite. The road towards an estimate of the $O(1 / N)$ corrections to the Flory exponent is then outlined.

The next section is devoted to the zero dimensional "toy model" and section 3 presents the computation of the simplest self energy diagramm to order $1 / N$. Short conclusions are contained in section 4 .

\section{The toy model.}

We study the problem of a classical particle which moves in a one dimensional space and feels a potential which is the sum of a harmonic term and a random term. We concentrate on the static equilibrium properties (some aspects of the dynamics of such a particle in the presence of an external force are reviewed in [5]). Denoting by $\omega$ the position of the particle, the Hamiltonian is:

$$
H=\frac{\mu}{2} \omega^{2}+V(\omega)
$$

$V$ is a random potential with a Gaussian distribution characterized by its first two moments:

$$
\overline{V(\omega)}=0 \quad \overline{V(\omega) V\left(\omega^{\prime}\right)}=-\frac{g}{2(1-\gamma)}\left(\omega-\omega^{\prime}\right)^{2-2 \gamma}+W \equiv f\left(\omega-\omega^{\prime}\right)
$$

(Actually the average free energy depends trivially on $W$, as can be seen by subtracting from $V$ a sample dependent uniform Gaussian random potential $U(\omega)=U$, with $\bar{U}=0$ and $\overline{U^{2}}=W$. In the following we keep to $W=0$ ).

This problem is nothing but a very simple case of the general problem of a $D$ dimensional manifold in a $N+D$ dimensional space which was studied in [1], where here we have $D=0$ and $N=1$. The slightly more general case of a particle in a $N$ dimensional space can be studied similarly, but here we want to keep to the simplest non trivial case $N=1$. Always for the sake of simplicity we keep to the case where the correlations of the potential are long range, $\gamma<1$. This problem has already been introduced and studied $[6,12]$ in the case $\gamma=1 / 2$. This case, which corresponds to a Brownian random potential, is also of particular interest since it describes the asymptotic behaviour of a directed polymer in a random potential in $1+1$ dimensions [11]. Hereafter we shall compare the results of the Gaussian variational Ansatz with r.s.b. to some "exact" results on this simple problem. We first rederive some analytic results using scaling analysis, and then we state the form of the results of [1] in this case and compare. The physical meaning of r.s.b is discussed in subsection 2.4 . In a fourth subsection we compare the variational r.s.b. results to some numerical simulations.

2.1 SCALING RELATIONS, EXACT Results. - Let $\Xi$ be the average over the quenched random potential of the logarithm of the partition function at temperature $T=1 / \beta$ :

$$
\Xi=\overline{\log \int \mathrm{d} \omega \mathrm{e}^{-\beta\left[\mu \omega^{2} / 2+V(\omega)\right]}}
$$


We study the system for one fixed value of the exponent $\gamma$ characterizing the correlations of the disorder. A priori, $\Xi$ is a function of the three variables $\beta, \mu$ and $g$. For any positive numbers $\lambda, \lambda^{\prime}$, one can easily see that it satisfies:

$$
\begin{gathered}
\Xi(\beta, \mu, g)=\Xi\left(\frac{\beta}{\lambda}, \lambda \mu, \lambda^{2} g\right), \\
\Xi(\beta, \mu, g)=\Xi\left(\beta, \frac{\mu}{\lambda^{\prime}},\left(\lambda^{\prime}\right)^{\gamma-1} g\right)-\frac{1}{2} \log \left(\lambda^{\prime}\right) .
\end{gathered}
$$

These equations show that:

$$
\Xi(\beta, \mu, g)=\xi(t)-\frac{1}{2} \log (\beta \mu)
$$

where $\xi$ depends only on the reduced temperature $t$ defined as:

$$
t=\frac{1}{\beta} \mu^{\frac{1-\gamma}{1+\gamma}}\left(\gamma 2^{1-2 \gamma} \frac{\Gamma(3 / 2-\gamma)}{\Gamma(1 / 2)} g\right)^{-\frac{1}{1+\gamma}},
$$

(the $\gamma$ dependent constant has been chosen in such a way as to simplify the formulae below). This shows in particular that the low temperature limit $(\beta \rightarrow \infty)$ is equivalent to the limit where the quadratic part of the potential vanishes $(\mu \rightarrow 0)$ and to the strong disorder limit $(g \rightarrow \infty)$. As for the correlation functions, they scale as:

$$
\overline{\left\langle\omega^{k_{1}}\right\rangle \cdot\left\langle\omega^{k_{r}}>\right.}=(\beta \mu)^{-\frac{k_{1}+\ldots+k_{r}}{2}} C_{k_{1} \ldots k_{r}}(t) .
$$

These scaling relations allow for simple derivations of some well known results. Let us consider the quantity $\overline{\left\langle\omega^{2}\right\rangle}$, at low temperatures. For $\gamma=1 / 2$ it has been shown exactly [12] that $\overline{\left\langle\omega^{2}\right\rangle} \simeq_{\beta \rightarrow \infty}\left(g / \mu^{2}\right)^{2 / 3}$, a result which can also be derived from an Imry Ma argument [13]. To derive this we start from equation (8) and notice that, for fixed $\mu$ and $g$, and $\beta \rightarrow \infty$, $\left\langle\omega^{2}\right\rangle$ must be finite (neither zero nor divergent). Therefore $C_{2}(t) \sim_{t \rightarrow 0}$ const $t^{-1}$ This gives the desired result:

$$
\overline{\langle\omega\rangle^{2}} \simeq_{\beta \rightarrow \infty} c^{t}\left(\frac{g}{\mu^{2}}\right)^{\frac{1}{1+\gamma}}
$$

The same reasoning shows that $\overline{\left\langle\omega^{2 p}\right\rangle}$ scales as $\left(g / \mu^{2}\right)^{p /(1+\gamma)}$. The disconnected correlation functions also have similar scaling behaviours (for instance $\overline{\langle\omega\rangle^{2}}$ scales as $\left(g / \mu^{2}\right)^{1 /(1+\gamma)}$ ). But the connected correlation functions behave differently:

$$
\overline{\left\langle\omega^{2}>\right.}-\overline{\langle\omega\rangle^{2}}=\frac{1}{\beta \mu}, \overline{\left\langle(\omega-<\omega>)^{4}\right\rangle}=\frac{3}{\beta^{2} \mu^{2}}, \text { etc... }
$$

These identities have been derived in a more general case in [12]. Let us briefly reproduce the proof in the present simple model. One considers the modified Hamiltonian $\tilde{H}(\omega)=$ $H(\omega)-\lambda / \beta$. In the partition function the shift $\omega \rightarrow \omega+\lambda /(\beta \mu)$ leads to:

$$
\overline{\log Z[\lambda]}=\overline{\log Z[\lambda=0]}+\frac{\lambda^{2}}{2 \beta \mu},
$$

provided that the distribution of the random potential is translationally invariant, which is the case here. Equations (10) follow from differentiating (11) with respect to $\lambda$.

While equations (10) are certainly correct, one should keep in mind that they compute averaged (over the distribution of the samples) quantities, which are not necessarily typical 
ones. For instance defining the susceptibility for one sample: $\chi=\left\langle\omega^{2}\right\rangle-\langle\omega\rangle^{2}$, we shall see that $\bar{\chi}$ is quite different from the typical $\chi$ of one given sample, a situation which is analogous with what has been found in directed polymers $[11,14]$.

2.2 Perturbative approach. - As simple as they may look, it is not trivial to obtain the scaling relations (8) using usual field theory methods, in contrast with the equations (10) for the connected correlations. For instance it has been shown that perturbation theory $[8,7]$ or iteration methods [9] fail at low temperatures because they are unable to take into account the many metastable states of the system.

The failure of perturbation theory has been proven by Villain [7]. One can either expand the random potential in power series of $\omega$, or use the replica approach and carry a perturbation expansion on the replicated system. Let us briefly describe the content of this second approach. The average of the replicated partition function is:

$$
\overline{Z^{n}}=\int \prod_{a=1}^{n} \mathrm{~d} \omega_{a} \exp \left(-\frac{\beta \mu}{2} \sum_{a=1}^{n} \omega_{a}^{2}+\frac{\beta^{2}}{2} \sum_{a, b} f\left(\omega_{a}-\omega_{b}\right)\right) .
$$

where the function $f$ is the correlation function of the potential given in (2). One can think of two types of expansions. The first one is to expand $\overline{Z^{n}}$ in powers of $f$, which is basically a high temperature expansion. In the present situation where there is no phase transition, this seems safe. The second type of expansion is the one which is used in the field theory studies of higher dimensional systems. It amounts to expand $f$ in series, to keep the quadratic term $\left(\omega_{a}-\omega_{b}\right)^{2}$, and to treat all the higher order terms in perturbative series [10]. Villain has shown that, in the limit of temperature going to zero, the correlation is $\overline{\left\langle\omega^{2}\right\rangle}=2 f^{\prime}(0) / \mu^{2}$ to all orders in perturbation. This is clearly a wrong result which violates the exact scaling results derived above. Therefore usual perturbation theory fails, and it is easy to trace back the reason of this failure to the existence of many metastable positions at low temperatures.

2.3 VARIATIONAL METHOD IN REPLICA SPACE. - We shall not reproduce here in all details the computation using a Gaussian function with r.s.b., since there are special cases of those performed in [1]. We shall just sketch the method and give the results. The method of [1] consists in using a variational method with the following trial hamiltonian:

$$
H_{\sigma}=\frac{\mu}{2} \sum_{a} \omega_{a}^{2}-\frac{1}{2} \sum_{a, b} \sigma_{a b} \omega_{a} \omega_{b}
$$

where the $\sigma$ parameters are to be determined by the stationarity condition of the variational free energy. These conditions read:

$$
\begin{gathered}
\sigma_{a b}=\beta \frac{g}{2} \frac{\Gamma(3 / 2-\gamma)}{\Gamma(1 / 2)}(1 / 2)^{\gamma-1}\left(\frac{G_{a a}+G_{b b}-2 G_{a b}}{\beta}\right)^{-\gamma} \\
\sigma_{a a}=-\sum_{b(\neq a)} \sigma_{a b} \\
G_{a b}=\left[(\mu-\sigma)^{-1}\right]_{a b} .
\end{gathered}
$$

The replica symmetric solution of these equations is:

$$
\sigma_{a b}=\sigma(a \neq b) \quad G_{a b}=\delta_{a b} \tilde{G}+\left(1-\delta_{a b}\right) G
$$


with:

$$
\tilde{G}-G=\frac{1}{\mu}, G=\frac{\sigma}{\mu^{2}}, \sigma=\beta^{1+\gamma} \mu^{\gamma} g 2^{-\gamma}
$$

This leads to the following two point correlation function:

$$
\overline{\left\langle\omega^{2}\right\rangle}=\frac{\tilde{G}}{\beta}=\frac{1}{\beta_{\mu}}\left(1+\frac{1}{\gamma} t^{-(\gamma+1)}\right),
$$

(the connected correlation function satisfies (10) as it should). This result is physically incorrect: for fixed $\mu$ and $g$, one finds that the correlation function $\overline{\langle\omega\rangle^{2}}$ diverges at low temperatures, which is impossible.

As was shown in [1], the replica symmetry is spontaneously broken in this approximation, at low enough temperatures. Using the hierarchical r.s.b. Ansatz [15], the matrix $\sigma_{a b}$ becomes a function of a continuous parameter $u \in[0,1]$. From [1] one sees that this function has two very different behaviours depending on whether $t$ is above or below a "critical temperature" $t_{\mathrm{c}}=1$. For $t>1$, the replica symmetric solution is valid and $\sigma$ is a constant. For $t<1$ the function $\sigma(u)$ is no longer constant. The result is then:

$$
\sigma= \begin{cases}\frac{\mu}{\gamma} t^{-1}, & u<u_{1}, \\ A \frac{1+\gamma}{2 \gamma} u^{\frac{2 \gamma}{1-\gamma}}, & u_{1}<u<u_{2} \\ A \frac{1+\gamma}{2 \gamma} u_{2}^{\frac{1+\gamma}{1-\gamma}}=\frac{\mu}{\gamma} t^{-\frac{1+\gamma}{1-\gamma}}, & u_{2}<u\end{cases}
$$

where:

$$
A=\mu t^{-\frac{1+\gamma}{1-\gamma}}, \quad u_{1}=\frac{1+\gamma}{2} t, \quad u_{2}=\frac{1+\gamma}{2}
$$

For this solution the two point correlation function is equal to:

$$
\overline{\left\langle\omega^{2}\right\rangle}= \begin{cases}\frac{1}{\beta \mu}\left(1+\frac{1}{\gamma} t^{-(\gamma+1)}\right), & t>1 \\ \frac{1}{\beta \mu} \frac{1+\gamma}{\gamma} t^{-1} & t<1\end{cases}
$$

This result is certainly more sensible. We shall discuss it hence, and compare it to numerical simulations in section 2.5. For simplicity one may choose for instance $\mu=1$ and $g=\frac{\Gamma(1 / 2)}{\Gamma(3 / 2-\gamma)} 2^{2 \gamma-1} / \gamma$, so that $t$ is the usual temperature: $t=1 / \beta$. Then the r.s.b. solution predicts the behaviour:

$$
\overline{\left\langle\omega^{2}\right\rangle}= \begin{cases}t+\frac{1}{\gamma t^{\gamma}}, & t>1 \\ 1+\frac{1}{\gamma} & t<1\end{cases}
$$


2.4 Physical discussion. - As we have seen above, the variational method predicts a phase transition in this system at a critical temperature $t_{\mathrm{c}}=1$. This phase transition is clearly unphysical in a system with only one degree of freedom. It is therefore an artefact of the variational method, but in a sense this is a clever artefact. The reason can be traced back to the physical interpretation of the quadratic trial hamiltonian (13) which has been worked out in [1]. This trial hamiltonian corresponds to a certain choice of the probability distribution of $\omega$.

For the case of a replica symmetric solution this probability is built as follows:

- to each sample is associated an average position of the particle, $\omega_{0}$, which is a random number drawn with the Gaussian distribution

$$
P\left(\omega_{0}\right)=\frac{1}{\sqrt{2 \pi G / \beta}} \exp \left(-\frac{\omega_{0}^{2}}{2 G / \beta}\right) ;
$$

- the thermal fluctuations of the particle position for one given sample are also Gaussian:

$$
p(\omega)=\frac{1}{\sqrt{2 \pi(\tilde{G}-G) / \beta}} \exp \left(-\frac{\left(\omega-\omega_{0}\right)^{2}}{2(\tilde{G}-G) / \beta}\right)
$$

where $G$ and $\tilde{G}$ are given in (18). This is clearly a simple minded distribution which is unable to take into account the many metastable states at low temperatures.

The r.s.b. solution corresponds to a much more complicated distribution as described in [1]. In a nutshell, at one step the breaking of the Gaussian $p(\omega)$ is subdivided into a superposition of Gaussians, with centers which are scattered (again with a Gaussian distribution) around $\omega_{0}$, and varying relative weights. At each new breaking the previous Gaussians are subdivided into superpositions of subgaussians, and the final distribution is the continuum limit of this hieriarchical breaking process. This is able to represent some quite complicated distribution with many metastable states.

A critical discussion of the meaning of the Gaussian variational r.s.b. solution in terms of the probability distribution of the particle's position has been done in [16]. To summarize it briefly, let us mention two facts:

- The fact that this variational approach finds a fake phase transition is not a surprise: a simple case of this phenomenon can be found in a double well potential of the type $V(\omega)=-a \omega^{2}+\omega^{4}$. If one performs a variational analysis based on the quadratic Hamiltonian $H_{\mathrm{v}}=\left(\omega-\omega_{0}\right)^{2} /(2 \Delta)$, there is a "phase transition" at $a=a_{\mathrm{c}}$ : For $a<a_{\mathrm{c}}$ the best solution is $\omega_{0}=0$, while for $a>a_{\mathrm{c}}$ it is $\omega_{0}= \pm \omega^{*}(a)$. (The true solution should have a symmetric double peak structure, but this requires including the tunneling states between the two minima. For large $a$ the variational solution gives, nevertheless, a rather good description of the system).

- The problem can be rephrased as that of finding the best functional probability distribution (over the choices of samples) of the probability distribution which describes the thermal fluctuations of the particle's position in one sample [16]. The variational Gaussian r.s.b. method provides a large class of such functionals, which is able to encode some "shaky" distributions characteristic of a problem with many metastable states.

Though the Gausssian variational r.s.b. solution of the previous section is still a rather crude approximation, it possesses some very interesting properties which are not so easy to obtain in this problem. The solution has the correct scaling form $C_{2}(t) \sim_{t \rightarrow 0} t^{-1}$ at low temperatures, which ensures that the scaling behaviours (9) and (10) are correct. This is not trivial, since for instance the scaling (10) of the connected correlations (which govern the thermal fluctuations) is dominated by rare samples [11,14]. This behaviour is reproduced here correctly as can be shown for instance by computing $\overline{\left(\left\langle\omega^{2}\right\rangle-\langle\omega\rangle^{2}\right)^{k}}[1]$. 

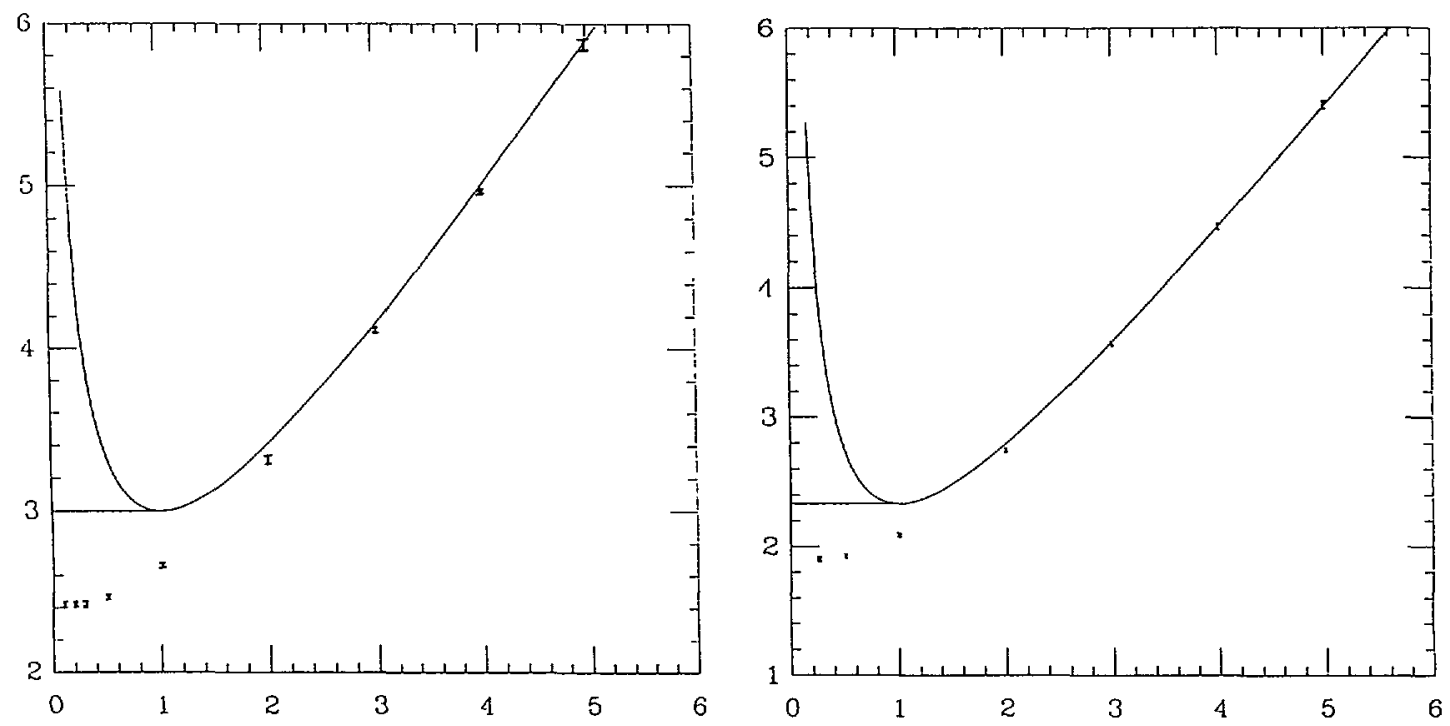

Fig.1. - Plot of the particle's position $\overline{\left\langle\omega^{2}\right\rangle}$ versus the reduced temperature $t$. The points are the results of the numerical simulation. The full lines are the replica symmetric (r.s.) and replica symmetry breaking (r.s.b.) Gaussian variational solutions. They coincide above $t=1$. Below $t=1$ the r.s. solution gives only nonsense, while the r.s.b. one approximates the gradual freezing by a phase transition. The exponent $\gamma$ which caracterizes the correlations of the potential is 0.5 for the left curve and 0.75 for the right curve.

2.5 Numerical Simulations. - We have performed some numerical simulations in order to test the prediction (23). We choose $\mu=1$ and $g=\frac{\Gamma(1 / 2)}{\Gamma(3 / 2-\gamma)} 2^{2 \gamma-1} / \gamma$. We discretize a suitable interval of the $\omega$ axis ( of length $\sim 20 / \sqrt{\beta}$ ) with typically of order $N=4000$ points and compute then $\overline{\left\langle\omega^{2}\right\rangle}$ exactly. The most extensive simulations, involving averaging over 25000 to 50000 samples, were done at $\gamma=1 / 2$ and $\gamma=.75$. The correlated potential is generated as follows: We generate $V_{0}, \ldots, V_{N}$ together with $V_{-N}, . . V_{-1}$ where $V_{-i} \equiv V_{i}$. This even and periodic function is obtained by fast Fourier transform from the Fourier components $\tilde{V}_{k}$. The $\tilde{V}_{k}$ are independent random variables of width $a_{k}$. The $a_{k}$ are chosen in such a way as to reproduce the correlations (2) exactly for the variables $V_{0}, . ., V_{N}$. An alternative solution for the case $\gamma=1 / 2$ is to generate $V_{i}=c^{t}\left(\sum_{j=0}^{i} h_{j}-\sum_{j=i}^{N} h_{j}\right)$, where $h_{i}$ are identically distributed random variables with a flat distribution. The results for $\overline{\left\langle\omega^{2}\right\rangle}$ are shown in figure 1, together with the prediction of the replica symmetric (r.s.) and r.s.b. Gaussian variational solutions. The r.s. and r.s.b. solutions coincide at high temperature and give the correct result. Actually we have checked that the high temperature expansion coincides with the r.s.b. solution at high $t$, with corrections of order $t^{(-1-2 \gamma)}$. The r.s. solution is totally wrong at low temperatures, as we already noticed. The r.s.b. solution is relatively good. It fails to describe the smooth freezing below $t=1 / \beta=1$ but approximates it by a phase transition. 


\section{The limit of large dimensions.}

The general problem of a $D$ dimensional directed manifold in a $N+D$ dimensional space, subjected to a random potential, is described by the Hamiltonian:

$$
H[\omega]=\frac{1}{2} \int \mathrm{d} x \sum_{\mu=1}^{D}\left(\frac{\partial \omega}{\partial x_{\mu}}\right)^{2}+\int \mathrm{d} x V(x, \omega(x)),
$$

where $\omega$ is a $N$ dimensional vector and $x$ is $D$ dimensional. The replica Gaussian variational method applied to this problem is exact when the space dimension $N$ goes to infinity (at $D$ and $\gamma$ fixed) [1]. The hierarchical r.s.b. Ansatz [15] then gives for the wandering exponent:

$$
\overline{\left\langle(\omega(x)-\omega(0))^{2}>\right.} \sim_{|x| \rightarrow \infty}|x|^{\frac{4-D}{(1+\gamma)}} .
$$

It would be particularly interesting to compute the corrections to this exponent to order $1 / N$. This should be possible using the usual methods of $1 / N$ expansion (see for instance $[18,17])$ for the replicated theory. The road is somewhat complicated by the fact that the zeroth order approximation has a spontaneous r.s.b.. Here we shall do only the first step in this direction, which is the computation of the simplest $O(1 / N)$ correction to the self energy. As in the usual case, this does not change the exponent and one needs to resum first of all the $O(1 / N)$ diagrams in order to get the logarithmic changes in the self energy which can be interpreted as a change of the wandering exponent to order $1 / N$.

Let us start from the large $N$ formulation of [1]. From (26) one gets a replicated Hamiltonian which describes a system of $n$ interacting manifolds ( $n$ the end $n$ goes to 0 ). For large $N$ it is convenient to introduce auxiliary fields:

$$
r_{a b}(x)=\frac{1}{N} \omega_{a}(x) \cdot \omega_{b}(x) \quad(a \leq b),
$$

together with the Lagrange multipliers $s_{a b}(x)$ which implement these constraints. This gives:

$$
\overline{Z^{n}}=\int \prod_{a \leq b}\left(\mathrm{~d}\left[r_{a b}\right] \mathrm{d}\left[s_{a b}\right]\right) \mathrm{e}^{N G[r, s]},
$$

with:

$$
\begin{aligned}
G[r, s] & =-\frac{\beta}{2} \sum_{a, b} \int \mathrm{d} x s_{a b}(x) r_{a b}(x)-\frac{\beta^{2}}{2} \int \mathrm{d} x \sum_{a, b} f\left(r_{a a}(x)+r_{b b}(x)-2 r_{a b}(x)\right)+S[s], \\
\mathrm{e}^{S[s]} & =\int \mathrm{d}\left[\omega_{1}\right] . . \mathrm{d}\left[\omega_{n}\right] \exp \left(-\frac{\beta}{2} \sum_{a, b} \int \mathrm{d} x \omega_{a}(x)\left(\left(-\nabla^{2}+\mu\right) \delta_{a b}-s_{a b}(x)\right) \omega_{b}(x)\right) .
\end{aligned}
$$

The infinite $N$ limit is worked out by solving the stationarity equations for $G[r, s]$, which is equivalent to using the variational method based on a Gaussian distribution. To go beyond, one expands $r$ and $s$ around the saddle point: $r_{a b}=r_{a b}^{\text {s.p. }}+\delta r_{a b}, s_{a b}=s_{a b}^{\text {s.p. }}+\delta s_{a b}$ and expands $G$ to second order in $\delta r, \delta s$. As explained in the Appendix I of [1], one can integrate easily over the fields $\delta r_{a b}(a \leq b)$ and $\delta s_{a a}$. After some work one finds the full order $1 / N$ correction to 


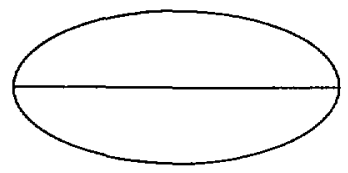

Fig.2. - The self energy diagram computed in the appendix.

the self energy:

$$
\delta \Sigma_{a b}(q)=\beta^{2} \sum_{c(\neq a), d(\neq b)} \int \frac{\mathrm{d}^{D} p}{(2 \pi)^{D}} K_{a c, d b}(p+q)\left(G_{c d}^{0}(p)-G_{a d}^{0}(p)-G_{c b}^{0}(p)+G_{a b}^{0}(p)\right),
$$

where $G_{a b}^{0}(p)=\left[\left(p^{2}-\Sigma\right)^{-1}\right]_{a b}$ is the infinite $N$ propagator computed in [1]. $K_{a c, d b}(p)$ is a $n(n-1) / 2 \times n(n-1) / 2$ matrix defined for all pairs of different replica indices. $K$ is the inverse of the matrix:

$$
\begin{aligned}
K_{a c, d b}^{-1}(k)= & N \frac{\delta_{(a c),(d b)}}{h_{a c}}-N \frac{\beta^{2}}{2} \int \frac{\mathrm{d}^{D} q}{(2 \pi)^{D}}\left(G_{a b}^{0}(q)+G_{c d}^{0}(q)-G_{a d}^{0}(q)-G_{b c}^{0}(q)\right), \\
& \left(G_{a b}^{0}(q+k)+G_{c d}^{0}(q+k)-G_{a d}^{0}(q+k)-G_{b c}^{0}(q+k)\right),
\end{aligned}
$$

with:

$$
h_{a b}=g \mid f^{\prime \prime}\left(\int \frac{\mathrm{d}^{D} q}{(2 \pi)^{D}}\left(G_{a a}^{0}(q)+G_{b b}^{0}(q)-2 G_{a b}^{0}(q)\right) \mid\right.
$$

A full computation of the order $(1 / N)$ corrections requires the inversion of $K^{-1}$ This is a complicated problem, which can probably be attacked using the methods developed in [19], but we shall leave this computation for future work. Here we make a simpler attempt by computing the diagram of figure 2. Its value is obtained by expanding $K$ to second order in $g$. The corresponding contribution to the self energy will be called $S_{a b}(q)$. It is given by:

$$
S_{a b}(q)=\frac{1}{N} \sum_{c(\neq a), \mathrm{d}(\neq b)} h_{a c} h_{b d} \int \frac{\mathrm{d}^{D} k_{1}}{(2 \pi)^{D}} \frac{\mathrm{d}^{D} k_{2}}{(2 \pi)^{D}} \frac{d^{D} k_{3}}{(2 \pi)^{D}} \delta\left(\sum_{i=1}^{3} k_{i}-q\right) B_{a b c d}\left(k_{1}\right) B_{a b c d}\left(k_{2}\right) B_{a b c d}\left(k_{3}\right)
$$

where:

$$
B_{a b c d}(k) \equiv G_{a b}^{0}(k)+G_{c d}^{0}(k)-G_{a d}^{0}(k)-G_{b c}^{0}(k) .
$$

One must evaluate $S_{a b}(q)$ using the infinite $N$ propagator of [1]. In the limit $n \rightarrow 0$ the pair of replica indices is turned into a continuous variable $u$ and the propagator becomes a function $G^{0}(k, u)$. The infinite $N$ self energy is a function $\Sigma(u)$ which scales at small $u$ like $\Sigma(u) \sim u^{(2 / \chi-1)}$ where $\chi$ is the exponent which governs the free energy fluctuations. The results of the detailed analysis of the long distance correlations performed in [1] can be recovered by simple scaling arguments. These amount to saying that the large distance limit of the physical propagator is dominated by the small $u$, small $k$ limit of $G(k, u)$, and that $u$ should be scaled as $k^{x}$ in this limit. Such a simple scaling analysis gives for $S(k)$ a behaviour in

$$
S(k) \sim_{k \rightarrow 0} k^{2(4-D)} k^{2 D} k^{-3(2+\chi)}
$$


In this formula, the first term comes from the factors $h_{a c} h_{b d}$ (using the scaling laws of [1]); the second term comes from the integrals over $k_{i}$ and the $\delta$ function, and the last term comes from the $B$ factors. Altogether this leads to:

$$
S(k) \sim_{k \rightarrow 0} k^{2-3 x},
$$

which is more singular than the infinite $N$ contribution: $S(k) \sim \Sigma(k) k^{-2 \chi}$. This simple scaling argument would mean that this single $O(1 / N)$ diagram changes the infinite $N$ exponents. However this turns out to be wrong. As can be seen in the full computation of $S$ described in the appendix, there is an extra cancellation among various terms in the sums over the replica indices $c$ and $d$, in equation (35). In fact the final scaling of $S(k)$ can still be deduced from a simple scaling analysis of (35), but using the fact that the sums over the two extra replica indices $c, d$ basically correspond to two extra integrations over internal " $u$ " type variables, which provide a new factor of $k^{2 \chi}$ needed to have $S$ scale in the same way as $\Sigma$.

This is a particularly miraculous cancellation if one considers the fact that several contributions to (35), like the term $c=b, d=a$ do not involve any integral over internal variables, and diverge individually like (38). It seems that such cancellations are frequent in the computation of loop diagrams in theories with replica symmetry breaking [20]. It would be very interesting to understand their origin. The final result for $S$ corresponds to the usual behaviour of the $1 / N$ correction of the critical exponents: In the usual $\left(\phi^{2}\right)^{2}$ theory this single diagram does not change the exponent, but one needs first to resum all the bubble diagrams in order to get the corrections to order $1 / N$ to the exponent. This could well be also the case here.

\section{Acknowledgments.}

We thank C. De Dominicis and I. Kondor for interesting discussions.

\section{Appendix.}

We compute the self energy $S_{a b}(q)$ defined in (35). In the sum over replica indices $c$ and $d$, one must distinguish several cases depending on which indices are equal. We write

$$
S_{a b}(q)=\frac{1}{N} \sum_{i=1}^{5} S_{a b}^{i}(q)
$$

where $S^{1}$ is the term $d=a, c=b, S^{2}$ is the term $d=c, S^{3}$ is the term $d=a, c \neq b, S^{4}$ is the term $d \neq a, c=b, S^{5}$ is the term where all the four replica indices are distinct. Introducing

$$
\begin{gathered}
g_{a b}^{i} \equiv G_{a a}^{0}\left(k_{i}\right)-G_{a b}^{0}\left(k_{i}\right) \text { and } \mathrm{d}(k) \equiv \frac{\mathrm{d}^{D} k_{1}}{(2 \pi)^{D}} \frac{\mathrm{d}^{D} k_{2}}{(2 \pi)^{D}} \frac{\mathrm{d}^{D} k_{3}}{(2 \pi)^{D}} \delta\left(\sum_{i=1}^{3} k_{i}-q\right), \text { we find } \\
S_{a b}^{1}(q)=-8 h_{a b}^{2} \int \mathrm{d}(k) g_{a b}^{1} g_{a b}^{2} g_{a b}^{3} \\
S_{a b}^{2}(q)=\sum_{a b c}^{\prime} h_{a c} h_{b c} \int \mathrm{d}(k)\left(g_{a c}^{1}+g_{b c}^{1}-g_{a b}^{1}\right)\left(g_{a c}^{2}+g_{b c}^{2}-g_{a b}^{2}\right)\left(g_{a c}^{3}+g_{b c}^{3}-g_{a b}^{3}\right) \\
S_{a b}^{3}(q)=\sum_{a b c}^{\prime} h_{a c} h_{b c} \int \mathrm{d}(k)\left(g_{b c}^{1}-g_{a b}^{1}-g_{a c}^{1}\right)\left(g_{b c}^{2}-g_{a b}^{2}-g_{a c}^{2}\right)\left(g_{b c}^{3}-g_{a b}^{3}-g_{a c}^{3}\right) \\
S_{a b}^{4}(q)=S_{b a}^{3}(q)
\end{gathered}
$$




$$
S_{a b}^{5}(q)=\sum_{a b c d}^{\prime} h_{a c} h_{b d} \int \mathrm{d}(k)\left(g_{a d}^{1}+g_{b c}^{1}-g_{a b}^{1}-g_{c d}^{1}\right)\left(g_{a d}^{2}+g_{b c}^{2}-g_{a b}^{2}-g_{c d}^{2}\right)\left(g_{a d}^{3}+g_{b c}^{3}-g_{a b}^{3}-g_{c d}^{3}\right)
$$

where the $\Sigma^{\prime}$ means that all the indices are distinct. In the limit $n \rightarrow 0$ the $n \times n$ matrices become functions of the internal parameter defined on the interval $[0,1]$. In terms of the functions $h$ and $g^{i}$ the above expressions read (denoting by $u$ the internal parameter associated with the pair of indices $(a b) 0$ :

$$
\begin{gathered}
S^{1}=-8 h(u)^{2} g^{1}(u) g^{2}(u) g^{3}(u) \\
S^{2}=-2 h(u) \int_{u}^{1} \mathrm{~d} v h(v) g^{1}(v) g^{2}(v) g^{3}(v)-h(u)^{2} u g^{1}(u) g^{2}(u) g^{3}(u) \\
-\int_{0}^{u} \mathrm{~d} v h(v)^{2}\left(2 g^{1}(v)-g^{1}(u)\right)\left(2 g^{2}(v)-g^{2}(u)\right)\left(2 g^{3}(v)-g^{3}(u)\right) \\
S^{3}+S^{4}=2 h(u) \int_{u}^{1} \mathrm{~d} v h(v) g^{1}(v) g^{2}(v) g^{3}(v)+2 h(u) \int_{0}^{u} \mathrm{~d} v h(v) g^{1}(u) g^{2}(u) g^{3}(u) \\
+2 h(u)^{2} u g^{1}(u) g^{2}(u) g^{3}(u)+2 h(u)^{2} \int_{u}^{1} \mathrm{~d} v\left(2 g^{1}(u)-g^{1}(v)\right)\left(2 g^{2}(u)-g^{2}(v)\right)\left(2 g^{3}(u)-g^{3}(v)\right) \\
S^{5}=2 \int_{u}^{1} \mathrm{~d} v \int_{0}^{u} \mathrm{~d} w h(u) h(v)\left(g^{1}(w)-g^{1}(u)\right)\left(g^{2}(w)-g^{2}(u)\right)\left(g^{3}(w)-g^{3}(u)\right) \\
+4 \int_{u}^{1} \mathrm{~d} v \int_{v}^{1} \mathrm{~d} w h(u) h(v)\left(g^{1}(w)-g^{1}(v)\right)\left(g^{2}(w)-g^{2}(v)\right)\left(g^{3}(w)-g^{3}(v)\right) \\
+\int_{u}^{1} \mathrm{~d} v \int_{u}^{1} \mathrm{~d} w h(u)^{2}\left(g^{1}(v)+g^{1}(w)-2 g^{1}(u)\right)\left(g^{2}(v)+g^{2}(w)-2 g^{2}(u)\right)\left(g^{3}(v)+g^{3}(w)-2 g^{3}(u)\right) \\
+2 \int_{0}^{u} \mathrm{~d} w \int_{0}^{w} \mathrm{~d} v h(v) h(w)\left(g^{1}(w)-g^{1}(u)\right)\left(g^{2}(w)-g^{2}(u)\right)\left(g^{3}(w)-g^{3}(u)\right) \\
+2 u h(u)^{2} \int_{u}^{1} \mathrm{~d} v\left(g^{1}(v)-g^{1}(u)\right)\left(g^{2}(v)-g^{2}(u)\right)\left(g^{3}(v)-g^{3}(u)\right) \\
+\int_{0}^{u} \mathrm{~d} v v h(v)^{2}\left(g^{1}(v)-g^{1}(u)\right)\left(g^{2}(v)-g^{2}(u)\right)\left(g^{3}(v)-g^{3}(u)\right) \\
+\int_{0}^{u} \mathrm{~d} v \int_{v}^{1} \mathrm{~d} w h(v)^{2}\left(2 g^{1}(v)-g^{1}(u)-g^{1}(w)\right)\left(2 g^{2}(v)-g^{2}(u)-g^{2}(w)\right)\left(2 g^{3}(v)-g^{3}(u)-g^{3}(w)\right) \\
+u \int_{u}^{1} \mathrm{~d} v h(u)^{2}\left(g^{1}(u)-g^{1}(v)\right)\left(g^{2}(u)-g^{2}(v)\right)\left(g^{3}(u)-g^{3}(v)\right)
\end{gathered}
$$

where the various contributions to each $S^{i}$ come from the various way of organizing the replica indices on an ultrametric tree. Adding up all these contributions, one finds the result announced in the text about the scaling of $S$ at small $u$ and small $q$. For instance the only term involving no integral over internal indices is:

$$
S(q, u) \ni \frac{1}{N} \int \mathrm{d}(k) h(u)^{2}(-7) g^{1}(u) g^{2}(u) g^{3}(u)
$$

The various terms containing $h(u)^{2} u^{\alpha} g^{1}(u) g^{2}(u) g^{3}(u)$ with $\alpha=0$ or $\alpha=1$, in the above expressions for $S^{i}$, cancel, leaving only the term $\alpha=2$ in the final sum. Similar cancellations occur in all the other dangerous terms. 


\section{References}

[1] Mézard M. and Parisi G., J. Phys. A 23 (1990) L1229; J. Phys. I France 1 (1991) 809.

[2] Bouchaud J.P., Mézard M. and Yedidia J.S., Phys. Rev. Lett. (1991) 3840; preprint LPTENS $92 / 3$.

[3] Bouchaud J.P. and Georges A., preprint LPTENS 92/4.

[4] Mézard M. and Young A.P., Europhys. Lett. 18 (1992) 653.

[5] Bouchaud J.P. and Georges A., Phys. Rep. 195 (1990) 127.

[6] Villain J., Semeria B., Lanon F. and Billard L., J.Phys. C16 (1983) 2588.

[7] Villain J. J. Phys. A 21 (1988) L1099.

[8] Engel A., J. Phys. Lett. France 46 (1985) L409.

[9] Vilain J, and Séméria B. J. Phys. Lett. France 44 (1983) L889.

[10] Physically the function $f$ should be regularized at small arguments, and in principle it can thus be expanded. Formula (2) actually refers to the asymptotic behaviour of $f$ at large arguments.

[11] Parisi G., J. Phys. France 51 (1990) 1595.

[12] Brezin E., Orland H., Shultz, Villain J., J.Stat.Phys. 51 (1988) 1.

[13] Imry Y. and Ma S.-k., Phys. Rev. Lett. 35 (1975) 1399.

[14] Mézard M., J. Phys. France 51 (1990) 1831.

[15] For a review see Mézard M., Parisi G. and Virasoro M.A., Spin glass theory and beyond (World Scientific, 1987).

[16] Mézard M. and Parisi G., A variational approach to directed polymers, Rome University preprint (1992).

[17] Parisi G., Statistical field theory (Addison, Wesley, 1988).

[18] Ma S.K., Phase transitions and critical phenomena, vol.6, C.Domb and M.S. Green Eds. (Academic press, 1976).

[19] Kondor I. and De Dominicis C., Europhys. Lett. 2 (1986) 617.

[20] De Dominicis C. and Kondor I., private conversation. 